\title{
Síndrome de Lemmel: ictericia obstructiva secundaria a divertículo duodenal yuxtapapilar. Reporte de un caso
}

\author{
Oliver R. Ramírez-Guerrero ${ }^{1 *}$ y Ma. Andrea Peñaloza-Posada² \\ ${ }^{1}$ Departamento de Cirugía General, Hospital General de México Dr. Eduardo Liceaga, Ciudad de México; ${ }^{2}$ Departamento de Endoscopia \\ Gastrointestinal, Hospital Regional de Alta Especialidad Ixtapaluca, Edo. Méx. México
}

\begin{abstract}
Resumen
El síndrome de Lemmel es una entidad poco frecuente que se caracteriza por la presencia de ictericia obstructiva secundaria a la presencia de un divertículo duodenal periampular. Los periampulares son los más comunes, se presentan en un 75\%; de estos, los que se localizan a 2-3 cm del ámpula de Vater se conocen como divertículos yuxtapapilares y la mayoría son extraluminales y adquiridos. Actualmente el diagnóstico se realiza utilizando colangiopancreatografía retrógrada endoscópica o ultrasonografía endoscópica, ya que con ambas se logra confirmar el diagnóstico. La esfinterotomía endoscópica y colocación de prótesis biliar es el tratamiento de elección en caso de complicación biliar o pancreática. Reportamos el caso de una paciente de 67 años con síndrome de Lemmel.
\end{abstract}

Palabras clave: Ictericia. Divertículo duodenal. CPRE. Hiperbilirubinemia. Síndrome de Lemmel.

\section{Lemmel Syndrome: obstructive jaundice secondary to juxtapapillary duodenal diverticulum. A case report}

\begin{abstract}
Lemmel syndrome is a rare entity, characterized by the presence of obstructive jaundice secondary to the presence of a periampular duodenal diverticulum. Periampular are the most common, in 75\%; of these, those located 2-3 cm from Vater's ampulla are known as juxtapapillary diverticula, most of which are extraluminal and acquired. Currently the diagnosis is made using endoscopic retrograde cholangiopancreatography or endoscopic ultrasonography, since both can confirm the diagnosis. Endoscopic sphincterotomy and biliary stent placement is the treatment of choice in case of biliary or pancreatic complications. We report the case of a 67-year-old patient with Lemmel syndrome.
\end{abstract}

Key words: Jaundice. Duodenal diverticulum. ERCP. Hyperbilirubinemia. Lemmel syndrome.

\section{Introducción}

El síndrome de Lemmel es una patología infrecuente, descrita en $1934^{1}$. Se define como ictericia obstructiva por divertículo duodenal periampular en ausencia de coledocolitiasis o neoplasia.
La segunda porción duodenal es la localización más frecuente, los divertículos periampulares están presentes en un $75 \%$; de estos, los que se localizan a $2-3 \mathrm{~cm}$ del ámpula de Vater se conocen como yuxtapapilares $^{2,3}$. Se presentan en entre el 5 y el $10 \%$ de la
Correspondencia:

*Oliver R. Ramírez-Guerrero

E-mail: oliverrenerg@ hotmail.com
Fecha de recepción: 24-07-2019

Fecha de aceptación: 17-04-2020

DOI: 10.24875/END.20000026

0188-9893/O 2020. Asociación Mexicana de Endoscopia Gastrointestinal y Colegio de Profesionistas, A.C. Publicado por Permanyer. Éste es un
artículo open access bajo la licencia CC BY-NC-ND (http://creativecommons.org/licenses/by-nc-nd/4.0/).

Disponible en internet: 00-00-2020

Endoscopia. 2020;32(2):65-68 www.endoscopia-ameg.com 
población general y su prevalencia incrementa con la edad. En México no existe registro epidemiológico de incidencia de divertículos duodenales y menos de este síndrome ${ }^{4}$.

Las manifestaciones clínicas son: ictericia, dolor abdominal, esteatorrea, obstrucción intestinal, diverticulitis y patología de la vía biliar (ictericia obstructiva, colangitis y pancreatitis) ${ }^{2}$. La coledocolitiasis ocurre con una incidencia del 20 al $40 \%$ en pacientes con divertículos periampulares. El $41 \%$ de los pacientes con divertículos yuxtapapilares y síntomas secundarios a obstrucción biliar tiene estudios de imagen normales ${ }^{5}$.

En los casos asintomáticos el tratamiento no está justificado, puesto que el desarrollo de una complicación es muy bajo. La esfinterotomía y/o colocación endoscópica de stent es el tratamiento de elección en caso de complicación biliar o pancreática. La cirugía se reserva ante el fracaso endoscópico. Actualmente no existe consenso sobre la técnica quirúrgica que realizar. La diverticulectomía simple se asocia a una alta morbimortalidad debido al riesgo de lesión del conducto biliopancreático, por ello es mandatorio localizar la ampolla de Vater mediante duodenectomía o de forma anterógrada a través del conducto cístico por coledocotomía. Asociar una coledocoyeyunostomía disminuye el riesgo de lesión. Otros autores sugieren añadir una derivación gastroentérica, sobre todo si hay inflamación local o riesgo de perforación diverticular. Se han descrito también otras opciones como la inversión diverticular, la esfinteroplastia transduodenal o la duodenopancreatectomía. Ninguna opción quirúrgica está exenta de complicaciones, por lo que el tratamiento se debe individualizar según las características del paciente, la localización del divertículo y la experiencia del centro ${ }^{6,7}$.

\section{Caso clínico}

Mujer de 67 años de edad, diabética e hipertensa. Colecistectomía abierta en 2018. Inicia tres días previos a su ingreso (marzo de 2019) con fiebre de $38.5^{\circ} \mathrm{C}$, dolor abdominal tipo cólico en cuadrante superior derecho, irradiado a epigastrio, emesis y evacuaciones diarreicas. A su ingreso con tensión arterial 49/26 $\mathrm{mmHg}$, frecuencia cardiaca $110 \mathrm{lpm}$, frecuencia respiratoria $25 \mathrm{rpm}$ y temperatura $38{ }^{\circ} \mathrm{C}$. Desorientada en las tres esferas, ictericia de escleras y tegumentos, exploración cardiopulmonar sin alteraciones, abdomen con peristalsis disminuida, dolor en flanco y fosa iliaca derecha, sin datos de irritación peritoneal. Leucocitosis $(20,900)$, trombocitopenia severa $(16,000)$,

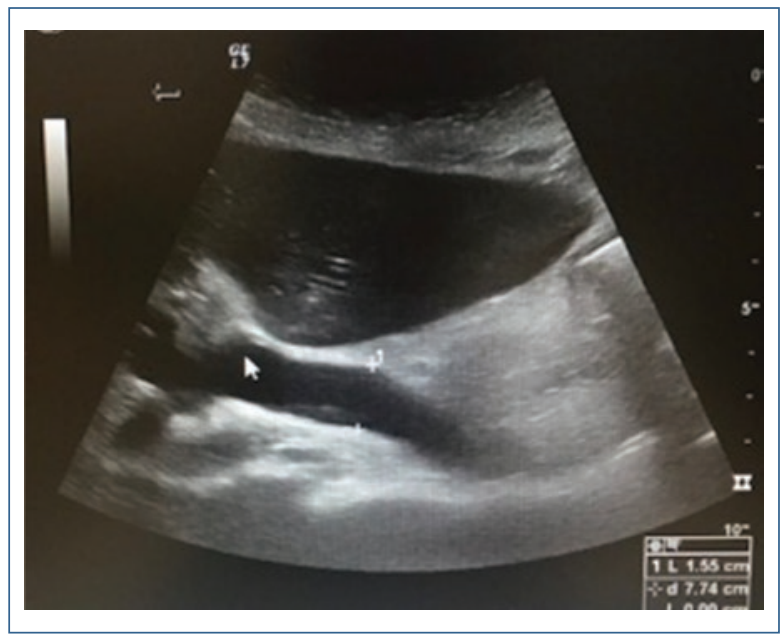

Figura 1. Colédoco dilatado en todo su trayecto de $15.5 \mathrm{~mm}$ de diámetro. Vesícula biliar ausente.

hipoglucemia (21 mg/dl), creatinina 4.8 , desequilibrio hidroelectrolítico, hiperbilirrubinemia a expensas de directa y transaminasemia, con colangitis severa (grado III Tokio). Ultrasonido: colédoco dilatado, $15.5 \mathrm{~mm}$ (Fig. 1).

Se inicia reanimación hídrica y vasopresores, ingresa a drenaje de la vía biliar por colangiopancreatografía retrógrada endoscópica (CPRE). En la CPRE: salida de detritus y pus, dilatación de la vía biliar intrahepática y extrahepática, fístula coledocoduodenal y divertículo yuxtapapilar. Se realiza lavado, barrido de detritus y pus con balón y colocación de prótesis. No se realiza esfinterotomía por trombocitopenia (Figs. 2 y 3).

La paciente ingresa a la unidad de terapia intensiva para antibioticoterapia endovenosa y apoyo aminérgico, con mejoría a las 48 horas. Egresa 10 días después de estancia hospitalaria.

\section{Discusión}

Este tipo de divertículos son asintomáticos en la mayoría de los casos y los que desarrollan sintomatología son los que se encuentran más cercanos al ámpula de Vater $(1 \mathrm{~cm})$. Pueden llegar a manifestarse como pseudotumores, diverticulitis, úlcera, perforación, sangrado de tubo digestivo alto, colangitis o pancreatitis crónica ${ }^{8}$.

Las manifestaciones clínicas más frecuentes relacionadas con este padecimiento son: ictericia, dolor abdominal, colangitis o mimetizando tumores periampulares. En general, la mayoría de los pacientes cursan no solo con ictericia a expensas de bilirrubina directa, 


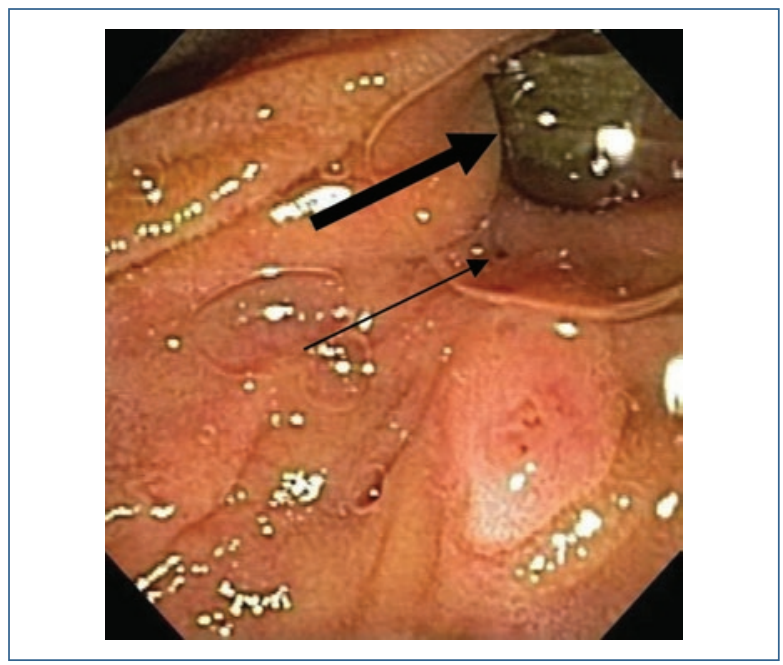

Figura 2. Se observa papila de tamaño normal, sin salida de bilis. En parte superior de la papila(flecha delgada) orificio de fístula colédoco duodenal. Divertículo yuxtapapilar con contenido alimentario en su interior (flecha gruesa).

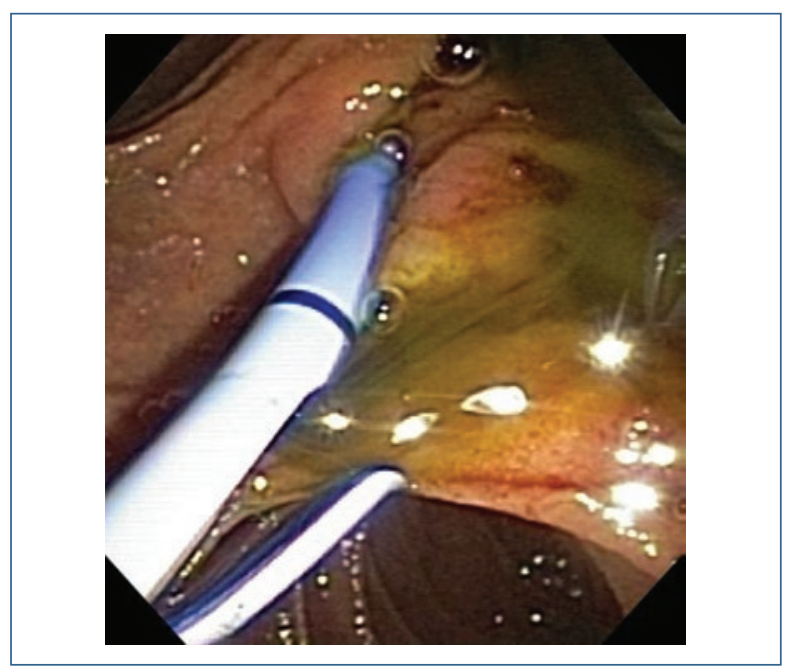

Figura 3. Colocación de endoprótesis plástica. Se observa abundante salida de pus y detritos.

sino también con otros síntomas, como dolor abdominal agudo o colangitis. Es característico que los pacientes presenten dolor epigástrico posprandial y sensación de llenura9.

La etiopatogenia del síndrome de Lemmel no está establecida y existen diversas etiologías, por ejemplo: irritación mecánica directa de los divertículos periampulares sobre el ámpula de Vater, provocando fibrosis papilar, así como por hipertensión secundaria al divertículo y/o disfunción del esfínter de Oddi y, por último, compresión mecánica del conducto biliar distal 0 ámpula ${ }^{10}$. Nuestra paciente probablemente tuvo irritación mecánica del esfínter y del colédoco distal ocasionando fístula hacia duodeno y colangitis.

La mejor manera de demostrar estos divertículos es utilizando el duodenoscopio. El diagnóstico no se realiza en estudios de imagen, se basa en la historia clínica y sospecha diagnóstica ${ }^{11}$. La CPRE está indicada para diagnóstico y tratamiento, aunque es invasiva y tiene complicaciones, es el estándar de oro para diagnóstico. La esfinterotomía endoscópica y colocación de endoprótesis biliar es el tratamiento de elección ${ }^{10}$. Los divertículos asintomáticos no requieren tratamiento ${ }^{12}$. El objetivo del tratamiento es aliviar la obstrucción biliopancreática, ya sea mediante endoscopia o cirugía. Actualmente el tratamiento endoscópico tiene una tasa alta de éxito ${ }^{2}$. La cirugía se reserva para complicaciones como perforación, hemorragia o colangitis de repetición. La diverticulectomía simple no se recomienda por alta morbimortalidad $(30 \%)^{13}$. Está descrita la inversión diverticular, esfinteroplastia transduodenal o duodenopancreatectomía. Ninguna opción quirúrgica está bien establecida, por esta razón el tratamiento se individualiza ${ }^{14}$. En este caso el tratamiento endoscópico con drenaje de la vía biliar fue el efectivo.

\section{Conclusiones}

El síndrome de Lemmel es una entidad caracterizada por una ictericia obstructiva, principalmente debida a un divertículo duodenal periampular; debe considerarse como un diagnóstico diferencial, cuyo manejo requiere un alto índice de sospecha y varios auxiliares diagnósticos y terapéuticas multidisciplinarias. El objetivo principal es asegurar el drenaje biliar evitando complicaciones pancreatobiliares que pueden resultar catastróficas.

En nuestro caso el tratamiento de elección incluyó CPRE sin esfinterotomía y colocación de stent biliar, asociado con un riesgo reducido de morbilidad y mortalidad.

\section{Agradecimientos}

El autor reconoce la cooperación de personas e instituciones que ayudaron a las investigaciones, así como a los que contribuyeron en la revisión y redacción del manuscrito del artículo. 


\section{Financiamiento}

La presente investigación no ha recibido ayudas específicas provenientes de agencias del sector público, sector comercial o entidades sin ánimo de lucro.

\section{Conflicto de intereses}

Los autores declaran no tener conflicto de intereses.

\section{Responsabilidades éticas}

Protección de personas y animales. Los autores declaran que para esta investigación no se han realizado experimentos en seres humanos ni en animales.

Confidencialidad de los datos. Los autores declaran que han seguido los protocolos de su centro de trabajo sobre la publicación de datos de pacientes.

Derecho a la privacidad y consentimiento informado. Los autores han obtenido el consentimiento informado de los pacientes y/o sujetos referidos en el artículo. Este documento obra en poder del autor de correspondencia.

\section{Bibliografía}

1. Lemmel G. Die klinische bedeutung der duodenal divertikel. Arch Verkrcht. 1934;56:59-70.

2. Rodriguez F, Polanía L, Evers S. A case of Lemmel's syndrome: A rare cause of non-neoplastic obstruction of the biliary tract. Rev Colomb Gastroenterol. 2017;32(1).

3. Desai K, Wermers J, Beteselassie N. Lemmel syndrome secondary to duodenal diverticulitis: a case report. Cureus. 2017;9:1066.

4. Carmona Agundez M, Lopez Guerra D, Fernandez Perez J, Blanco Fernandez G. Lemmel's syndrome: obstructive jaudince secondary to a duondenal diverticulum. Cir Esp. 2017;95(9):545-54.

5. Musumba C, Britton E, Smart H. A man presenting with severe postprandial epigastric pain, jaundice, and pyrexia: more than the usual suspects? Gastroenterology. 2013;144(2):274-469.

6. Mathis KL, Farley DR. Operative management of symptomatic duodenal diverticula. Am J Surg. 2007;193:305-8. Discussion 308-9.

7. Schnueriger B, Vorburger SA, Banz VM, Schoepfer AM, Candidas D. Diagnosis and management of the symptomatic duodenal diverticulum: A case series and short review of the literature. J Gastrointest Surg. 2008;27:105-9.

8. Mendez CJ, Medina AL, Diaz RJ, Flores RD, Vidrio DE, Jhybran TB, et al. Cholangitis secondary to Lemmel syndrome. Case report. Arch Clin Gastroenterol. 2016;2:77-9.

9. Wang YF, Liu KL, Yang JC. A rare cause of obstructive jaudince. Clin Gatroenterol Hepatol. 2013;11:A31.

10. Rouet J, Gaujoux S, Ronot M, Palazzo M, Cauchy F, Vilgrain V, et al. Lemmel's syndrome as rare cause of obstructive jaudince. Clin Res Hepatol Gastroenterol. 2012;36(6):628-31.

11. Kang HS, Hyun JJ, Kim SY, Jung SW, Koo JS, Yim HJ, et al. Lemmel's syndrome, an unusual cause of abdominal pain and jaundice by impacted intradiverticular enterolith: Case report. J Korean Med Sci. 2014;29(6):874-8.

12. Beisani M, Espin F, Dopazo C, Quiroga S, Charco R. Manejo terapéutico del divertículo duodenal yuxtapapilar. Cir Esp. 2013;91:463-5.

13. Tobin R, Barry N, Foley NM, Cooke F. A giant duodenal diverticulum causing Lemmel syndrome. J Surg Case Rep. 2018;2018(10):rjy263.

14. Melo Muñoz DK, Sánchez Sotelo VM, Dávila Jaimes GÁ. Ictericia obstructiva secundaria a síndrome de Lemmel. Reporte de caso. Revista Mexicana de Cirugía Endoscópica. 2018;19(3):110-2. 